\title{
La crítica de George Berkeley al representacionalismo de John Locke
}

\author{
Alberto Oya ${ }^{1}$
}

Recibido: 18 de marzo de 2016 / Aceptado: 04 de noviembre de 2017

Resumen. En su Tratado sobre los principios del conocimiento humano, George Berkeley ofrece una serie de argumentos cuyo objetivo es criticar la tesis materialista. Mi propósito en este artículo es reconstruir y analizar en detalle estos argumentos. Dado que la crítica de Berkeley al materialismo es, fundamentalmente, una crítica al materialismo representacionalista de John Locke, empezaré este artículo explicando cuáles son las ideas básicas de la propuesta de Locke.

Palabras clave: cualidades primarias; cualidades secundarias; George Berkeley; inmaterialismo; John Locke; materialismo; representacionalismo.

\section{[en] George Berkeley's criticism to John Locke's representationalism}

\begin{abstract}
In his Treatise concerning the Principles of Human Knowledge, George Berkeley offers some arguments in order to criticize the materialist position. My aim in this paper is to expound and analyze in detail these arguments. Given that Berkeley's criticism to materialism is, at the end, a criticism to John Locke's representationalism, I will begin this paper explaining which are the core ideas of Locke's proposal.
\end{abstract}

Keywords: George Berkeley; immaterialism; John Locke; materialism; primary qualities; representationalism; secondary qualities.

Sumario. 1. El contexto filosófico del inmaterialismo berkelyano; 1.1. El representacionalismo de John Locke; 1.2. La propuesta inmaterialista de Berkeley en su contexto histórico; 2. La crítica de Berkeley al representacionalismo; 2.1. El principio de semejanza; 2.2. Crítica a la distinción entre cualidades primarias y cualidades secundarias; 2.3. La expresión 'sustancia material' carece de significado; 2.4. El representacionalismo conduce al escepticismo; 2.5. El representacionalismo no es la mejor explicación; 3. Conclusión.

Cómo citar: Oya, A. (2018): "La crítica de George Berkeley al representacionalismo de John Locke", en Revista Anales del Seminario de Historia de la Filosofía 35 (1), 109-126.

$1 \quad$ Universitat de Girona

alberto.oya.marquez@gmail.com

ORCID: 0000-0002-3996-5366 


\section{El contexto filosófico del inmaterialismo berkelyano}

La propuesta inmaterialista de George Berkeley se encuentra estrechamente ligada al contexto filosófico de su época. De hecho, como quedará claro más adelante, el punto de partida del inmaterialismo berkelyano no parece ser otro que la llamada concepción causal clásica del conocimiento perceptivo y el representacionalismo de John Locke.

La concepción causal clásica del conocimiento perceptivo se caracteriza, en primer lugar, por definir la percepción como una relación causal entre el sujeto y el mundo. De aquí que se distinga la causa u origen de la percepción, identificada con un determinado objeto del mundo, del efecto o contenido de la percepción, identificado con la imagen interna que el sujeto perceptor conoce por introspección.

Otra de las características básicas de esta concepción es que establece que la percepción no puede ser una relación epistémica entre el sujeto y el mundo -al menos de manera inmediata. Esto es así porque el sujeto sólo conoce, por introspección, su imagen interna, que se presupone que es causada por el estímulo externo, pero no el objeto externo. Dentro de este paradigma, la única manera de que la percepción sea una relación epistémica entre el sujeto y el mundo, aunque de manera mediata, es postular el representacionalismo -es decir: afirmar que las imágenes internas del sujeto representan, ya sea de manera total o parcial, el objeto externo.

Veamos un ejemplo. De acuerdo con la concepción causal clásica del conocimiento perceptivo, cuando un individuo cualquiera, llamémosle $P$, está percibiendo un árbol concreto, digámosle $x$, se dan tres procesos simultáneos y diferentes: (i) una relación causal entre $x$, que es externo a la mente de $P$, y la imagen interna de $x$ que tiene $P$ en su mente, en la que $x$ es la causa de la imagen interna de $x \mathrm{y}$, por tanto, la segunda es el efecto de la primera; (ii) una relación epistémica inmediata, por introspección, entre $P$ y su imagen interna de $x$; (iii) una relación epistémica mediata, mediante las ideas internas de $P$, entre $P$ y $x$.

Laconcepción causal clásica del conocimiento perceptivo es el marco interpretativo predominante en la epistemología moderna, ya que es aceptada, des de Galileo, por los principales pensadores de la época, tanto racionalistas como empiristas. ${ }^{2} \mathrm{La}$ hipótesis cartesiana del genio maligno, por ejemplo, presupone que no mantenemos una relación epistémica inmediata con el mundo externo. De hecho, el objetivo de dicha hipótesis no es otro que mostrar que no hay ningún tipo de necesidad en el vínculo causal entre el estímulo externo y la imagen interna que se cree se da en todo proceso perceptivo. En otras palabras, y siguiendo el ejemplo anterior, Descartes está argumentando que existe la posibilidad de que percibiéramos una imagen interna de árbol sin que dicha imagen fuera producida por un árbol externo sino por «[...] cierto genio maligno, no menos artero y engañador que poderoso, el cual ha usado de

\footnotetext{
A pesar de que esta concepción fue la predominante en la época, cabe mencionar que no todos los filósofos la adoptaron. De entre todos ellos, el caso más conocido sea, seguramente, Thomas Reid (1710-1796). En su Investigación sobre la mente humana en los principios de sentido común (1734), Reid rechazó la afirmación de que el sujeto perceptor mantiene únicamente una relación epistémica, por introspección, con sus ideas o imágenes internas, en tanto que dicha visión contradecía lo que él consideraba la opinión del sentido común; i.e., que mantenemos una relación epistémica directa con los objetos externos. Excede los propósitos de este artículo el ofrecer un análisis pormenorizado de la argumentación ofrecida por Reid. Si el lector está interesado en una exposición sistemática de la propuesta filosófica de Reid recomiendo la lectura de: González de Luna, Eduardo M. Filosofía del sentido común. México: Universidad Nacional Autónoma de México, 2004 (especialmente el capítulo 2: "La filosofía del sentido común de Thomas Reid", pp. 37-56).
} 
toda su industria para engañarme», ${ }^{3} \mathrm{y}$ que, simplemente, no hubiera, de hecho, nada parecido a árboles en el mundo externo.

\subsection{El representacionalismo de John Locke}

Con el Ensayo sobre el entendimiento humano (1690) de John Locke (1632-1704), el proyecto representacionalista adopta una forma mucho más compleja y refinada. La propuesta de Locke tiene dos pilares fundamentales: en primer lugar, la distinción entre cualidades primarias y cualidades secundarias; y, en segundo lugar, la postura materialista, que tiene su máxima expresión en el recurso a la llamada 'sustancia material'.

La noción de cualidad y, dentro de esta, la distinción entre cualidades primarias y cualidades secundarias, es introducida por John Locke en el capítulo octavo del libro segundo de su Ensayo. Si las ideas son «[...] todo aquello que la mente percibe en sí misma, o todo aquello que es el objeto inmediato de percepción, de pensamiento, o de entendimiento», ${ }^{4}$ las cualidades, en cambio, son capacidades o potencias de un objeto de producir en nosotros cierta idea. El autor ejemplifica esta distinción como sigue:

[...] una bola de nieve tiene el poder de producir en nosotros las ideas de blanco, frío, y redondo, esos poderes de producir en nosotros esas ideas, en cuanto que están en la bola de nieve, los llamo cualidades; y en cuanto que son sensaciones o percepciones en nuestro entendimiento, los llamo ideas [...]. ${ }^{5}$

Pero no todas las cualidades son iguales y, por ello, dice Locke, debemos distinguir las cualidades primarias de las cualidades secundarias. Las primarias son aquellas cualidades esenciales o inalienables al cuerpo en qué se encuentran, pues éste «en todas las alteraciones y cambios que sufra [...] las conserva constantemente». ${ }^{6}$ Locke considera como cualidades primarias aquellas que producen en nosotros las ideas de solidez, extensión, figura, movimiento o reposo, y número. Las secundarias, en cambio, aunque Locke las llame 'cualidades', no son realmente cualidades, pues no se encuentran en ningún sitio fuera de la mente y no son predicados de ningún objeto externo, sino sólo «poderes de producir en nosotros varias sensaciones por medio de sus cualidades primarias». ${ }^{7}$ Es decir: por 'cualidades secundarias' debemos entender la capacidad o poder de los objetos externos de producir en nosotros aquellas ideas de cualidades, cualidades que no son presentes en los objetos externos que las causan, como es el caso de los colores, olores, sonidos y sabores.

3 Descartes, René. Meditaciones metafisicas con objeciones y respuestas. Oviedo: KRK Ediciones, 2005, pp. $137-138$.

4 Locke, John. An Essay concerning Human Understanding. Hong Kong: Oxford University Press, 1990. II, viii, $\S 8$. La traducción al castellano es mía; la cita original dice así: «[...] whatsoever the Mind perceives in it self, or is the immediate object of Perception, Thought, or Understanding».

5 Locke, John. op. cit. II, viii, §8. La cursiva es de Locke. La traducción al castellano es mía; la cita original dice así: «[...] a Snow-ball having the power to produce in us the Ideas of White, Cold, and Round, the Powers to produce those Ideas in us, as they are in the Snow-ball, I call Qualities; and as they are Sensations, or Perceptions, in our Understanding, I call them Ideas $[\ldots]$...

6 Locke, John. op. cit. II, viii, §9. La traducción al castellano es mía; la cita original dice así: «such as in all the alterations and changes it suffers $[\ldots]$ it constantly keeps».

7 Locke, John. op. cit. II, viii, §10. La cursiva es de Locke. La traducción al castellano es mía; la cita original dice así: «Powers to produce various Sensations in us by their primary Qualities». 
Sin embargo, la diferencia principal no se encuentra en las propias cualidades pues ya hemos visto que, de hecho, sólo hay cualidades primarias- sino en el carácter representacional de las ideas de dichas cualidades. Así, Locke concluirá que las ideas de cualidades primarias representan fielmente las características del mundo externo, ${ }^{8}$ mientras que las ideas de cualidades secundarias no hacen tal cosa, en tanto que estas últimas se definen por la relación que establecen las cualidades primarias con los sujetos perceptores; más concretamente, por la extensión, número y volumen de los corpúsculos que configuran el objeto, así como por la relación física, ya sea por su movimiento o reposo, que establecen con los sujetos perceptores. Por ejemplo: percibimos un conjunto de ideas internas que son causadas por un objeto externo a nosotros - por ejemplo: una pelota-, y en nuestra mente se nos aparece la pelota como siendo redonda y azul; ${ }^{9}$ en este caso no podemos decir que la pelota es en sí misma azul, pero sí que podemos decir que es en sí misma redonda.

Por lo que refiere al carácter representacional de nuestras ideas de cualidades secundarias, el ejemplo propuesto por Locke es el siguiente:

En un sentido estricto, la propiedad de ser amarillo [«Yellowness», en el texto original] no está realmente en el oro, sino que es un poder del oro de producirnos esa idea en nuestros ojos, cuando éste se encuentra bajo la luz adecuada; y el calor, que no podemos dejar de lado de nuestra idea de sol, no es más real en el sol que el color blanco lo es en la cera. Ambos son poderes del sol, operando, mediante el movimiento y figura de sus insensibles partes, en el hombre, haciendo que éste tenga la idea de calor; y operando del mismo modo en la cera, haciendo que el hombre tenga la idea de blanco. ${ }^{10}$

La principal razón ofrecida por Locke a favor de la distinción entre cualidades primarias y cualidades secundarias es el que posteriormente ha sido conocido como el argumento de la relatividad de la percepción. Y es que, de acuerdo con Locke, gracias a la distinción entre cualidades primarias y secundarias podemos explicar, de una manera coherente, el porqué una misma corriente de agua nos puede llegar a parecer fría en una mano y caliente en la otra, y, sin embargo, porqué un objeto cualquiera, por ejemplo, una pelota, no nos puede parecer redondo en una mano y cuadrado en la otra. En palabras de Locke:

Entendiendo y diferenciando las ideas de este modo, podemos explicar cómo la misma corriente de agua, puede, al mismo tiempo, producir la idea de frío a una mano y la de calor en la otra. Es imposible que la misma corriente de agua sea fría y caliente al mismo tiempo, que es lo que tendría que suceder si esas ideas estuvieran realmente en ella. Pues si imaginamos que el calor, en cuanto está en nuestras manos, no es nada más que un cierto tipo y grado de movimiento en las minúsculas partículas de nuestros nervios o

8 Y con «representar fielmente» se está diciendo que las ideas de cualidades primarias se asemejan, literalmente, al mundo externo.

9 Es decir: nuestra idea compleja del objeto «pelota azul» está formada por una idea de cualidad primaria («redonda») y una idea de cualidad secundaria («azul»).

10 Locke, John. op. cit. II, xxiii, §10. La cursiva és de Locke. La traducción al castellano es mía; la cita original dice así: «For to speak truly, Yelowness is not actually in Gold; but is a Power in Gold, to produce that Idea in us by our Eyes, when placed in a due Light: and the Heat, which we cannot leave out of our Idea of the Sun, is no more really in the Sun, than the white Colour it introduces into Wax. These are both equally Powers in the Sun, operating, by the Motion and Figure of its insensible Parts, so on a Man, as to make him have the Idea of Heat; and so on Wax, as to make it capable to produce in a Man the Idea of White». 
espíritus animales, podremos entender por qué es posible que la misma agua pueda, al mismo tiempo, producir la sensación de calor en una mano y la de frío en otra; y, sin embargo, por qué una figura que ha producido la idea de redondo a una mano, nunca produzca la idea de cuadrado a la otra. ${ }^{11}$

Más adelante veremos con más detalle el uso que hace Locke del argumento de la relatividad de la percepción. Por el momento, lo importante ahora es hacer notar que lo que Locke está haciendo aquí es ofrecer una explicación de carácter materialista de cómo se originan, en nuestro entendimiento, nuestras ideas de percepción: los objetos externos desprenden un volumen de corpúsculos o partículas que, al entrar en contacto con nuestros órganos sensoriales, producen en nosotros las ideas de cualidades. En palabras de Locke:

[por lo que refiere a nuestras ideas de cualidades primarias] [...] dado que la extensión, figura, número y movimiento de los cuerpos de un tamaño observable pueden ser percibidos a distancia por la vista, es evidente que algunos cuerpos individualmente imperceptibles deben de venir de ellos a los ojos, comunicando así al cerebro cierto movimiento, el cual produce esas ideas que tenemos nosotros acerca de ellos. [...] [por lo que refiere a nuestras ideas de cualidades secundarias] Supongamos ahora que los diferentes movimientos y figuras, volumen y número de tales partículas, afectando a los diferentes órganos de nuestros sentidos, producen en nosotros esas diferentes sensaciones que tenemos acerca de los colores y los olores de los cuerpos -por ejemplo, que una violeta, por el impulso de esas partículas de materia insensibles, en su peculiar figura y volumen, y en sus diferentes grados y modificaciones de sus movimientos, cause que las ideas del color azul y del dulce aroma de esa flor se produzcan en nuestras mentes. ${ }^{12}$

Siguiendo el ejemplo propuesto anteriormente, podemos decir que, de acuerdo con Locke, cuando un individuo cualquiera está percibiendo un árbol concreto, se dan los siguientes procesos diferentes -y se presupone que también simultáneos-: (i) una relación causal y una relación de semejanza entre un conjunto de corpúsculos (que presentan cualidades primarias concretas) y las ideas internas de cualidades primarias que percibe el sujeto; (ii) una relación causal entre un conjunto de corpúsculos (que presentan cualidades primarias concretas, pero no cualidades

11 Locke, John. op. cit. II, viii, §21. La cursiva es de Locke. La traducción al castellano es mía; la cita original dice así: «Ideas being thus distinguished and understood, we may be able to give an Account, how the same Water, at the same time, may produce the Idea of Cold by one Hand, and of Heat by the other: Whereas it is impossible, that the same Water, if those Ideas were really in it, should at the same time be both Hot and Cold. For if we imagine Warmth, as it is in our Hands, to be nothing but a certain sort and degree of Motion in the minute Particles of our Nerves, or animal Spirits, we may understand, how it is possible, that the same Water may at the same time produce the Sensation of Heat in one Hand, and Cold in the other; which yet Figure never does, that never producing the Idea of a square by one Hand, which has produced the Idea of Globe by another».

12 Locke, John. op. cit. II, viii, §12-13. La cursiva es de Locke. La traducción al castellano es mía; la cita original dice así: «[...] since the Extension, Figure, Number, and Motion of Bodies of an observable bigness, may be perceived at a distance by the sight, "tis evident some singly imperceptible Bodies must come from them to the Eyes, and thereby convey to the Brain some Motion, which produces these Ideas, which we have of them in us. [...] Let us suppose at present, that the different Motions and Figures, Bulk, and Number of such Particles, affecting the several Organs of our Senses, produce in us those different Sensations, which we have from the Colours and Smells of Bodies, v.g. that a Violet, by the impulse of such insensible particles of matter of peculiar figures, and bulks, and in different degrees and modifications of their Motions, causes the Ideas of the blue Colour, and sweet Scent of that Flower to be produced in our Minds» 
secundarias) y las ideas internas de cualidades secundarias que percibe el sujeto; (iii) la unión, si puede llamarse así, de las ideas de cualidades primarias y de las ideas de cualidades secundarias en una sola idea -en este caso, en la «idea de árbol»-, que es con la que el individuo mantiene una relación epistémica inmediata; (iv) una relación epistémica mediata -mediante las ideas internas-y parcial-sólo sobre las cualidades primarias- entre el sujeto y el mundo.

Nótese que este tercer paso es algo que Locke no explicita, quizás porque es consciente de las dificultades que el mismo conlleva. Y el problema reside en cómo entender esta unión de las ideas de cualidades primarias y las ideas de cualidades secundarias. Parece que sólo tenemos dos opciones: (1.) es la mente del sujeto perceptor quien, una vez percibidas aisladamente, las une; (2.) las ideas de cualidades primarias y las ideas de cualidades secundarias son ya percibidas unidas. Si, por un lado, (1.) fuera cierto, entonces el sujeto perceptor debería de poder deshacer la unión que él mismo ha establecido $\mathrm{y}$, en consecuencia, debería de poder percibir aisladamente las ideas de cualidades, tanto primarias como secundarias. Esto, sin embargo, es falso: entendiendo las ideas como imágenes mentales, uno no puede llegar a percibir aisladamente una idea de cualidad, sea cual sea ésta -es decir: uno no puede formarse una imagen mental que tenga como contenido tan sólo «azul», «redondo», «cuadrado», etcétera. Esto es, precisamente, lo que Berkeley muestra con su crítica a las ideas abstractas, tal y como la desarrolla en la Introducción de su Tratado sobre los Principios del Conocimiento Humano. Y, por otro lado, si (2.) fuera cierto, ¿cómo podríamos distinguir lo que es una idea de cualidad primaria de lo que es una idea de cualidad secundaria?

Una vez hemos visto la distinción entre cualidades primarias y cualidades secundarias, es fácil de ver cuál es el papel de la llamada 'sustancia material' en la propuesta representacionalista de Locke. La sustancia material no es otra cosa, dice Locke, que

[...] tan sólo una suposición de no se sabe qué soporte de aquellas cualidades que son capaces de producir en nosotros ideas simples; cualidades que son comúnmente conocidas con el nombre de accidentes. ${ }^{13}$

Y de dicha sustancia material no tenemos ninguna idea positiva, sino sólo relativa -esto es: que es el soporte en el cual subsisten las cualidades primarias que causan nuestras ideas internas de percepción. La pregunta es, por tanto, cómo llegamos a esta idea relativa de sustancia material. La respuesta de Locke es que dicha idea es fruto de una inferencia, a partir de nuestra convicción que las cualidades primarias no pueden subsistir por sí mismas:

La idea, pues, que tenemos de aquello que damos el nombre general de sustancia no es otra cosa que el supuesto, aunque desconocido, soporte de esas cualidades, las cuales encontramos que existen e imaginamos que no pueden subsistir sine re substante, sin algo que las soporte. Y a este soporte es a lo que llamamos sustancia; que, de acuerdo con el verdadero significado de esta palabra, significa en castellano común [«plain English», en

13 Locke, John. op. cit. II, xxiii, §2. La cursiva és de Locke. La traducción al castellano es mía; la cita original dice así: «[...] only a Supposition of he knows not what support of such Qualities, which are capable of producing simple Ideas in us; which Qualities are commonly called Accidents». 
el texto original] que sustenta, o que está debajo [«standing under, or upholding», en el texto original]. ${ }^{14}$

\subsection{La propuesta inmaterialista de Berkeley en su contexto histórico}

Tan sólo veinte años después de la publicación del Ensayo sobre el entendimiento humano (1690), apareció el Tratado sobre los principios del conocimiento humano (1710), de la mano de George Berkeley (1685-1753), quien, partiendo de las tesis básicas del marco interpretativo de la epistemología moderna, llegará a una conclusión muy diferente a la que habían llegado los autores anteriores.

La idea básica de su propuesta puede ser resumida en la sentencia «esse est percipi aut percipere» («ser es percibir o ser percibido»); es decir: que sólo existe aquello percibido y aquello que percibe. Y dado que Berkeley acepta que los sujetos perceptores son de carácter espiritual o no material y que aquello que los sujetos perciben son ideas internas a ellos de carácter mental o no material, su propuesta supone negar la existencia de un mundo físico externo a los sujetos perceptores.

Esto no quiere decir, no obstante, que Berkeley considere que las ideas de percepción sean subjetivas, en el sentido de que dependan de, o sean causadas por, los propios sujetos que las perciben: lo que afirmará Berkeley es que las ideas de percepción tienen su causa en Dios, quien no es otra cosa que un espíritu perceptor todopoderoso, infinitamente sabio, y otros atributos similares. ${ }^{15}$

Para mostrar la validez de su «esse est percipi aut percipere», Berkeley lleva a cabo una doble argumentación. Realiza, en primer lugar, lo que podríamos considerar una argumentación de carácter crítico o reductivo, mediante la cual pretende mostrar, entre otras cuestiones, que la distinción entre cualidades primarias y cualidades secundarias defendida por Locke es falsa, que el representacionalismo conduce inevitablemente al escepticismo o que la noción de sustancia material de la que hablaba Locke carece de significado. La segunda argumentación que sigue Berkeley se aleja de este carácter más crítico y está constituida por tres argumentos que adoptan un carácter más positivo, los cuales pretenden demostrar la inconcebibilidad de un mundo externo de carácter físico. ${ }^{16}$ Dicho de manera más breve: en el primer caso Berkeley pretende probar que las propuestas anteriores de carácter materialista son indeseables o que carecen de contenido; en el segundo caso pretende, directamente, probar el inmaterialismo.

También es importante hacer notar que Berkeley en ningún momento niega el supuesto básico de la epistemología moderna que comenté unas líneas atrás -a saber: que el individuo sólo mantiene una relación epistémica inmediata, por introspección, con sus propias ideas internas, que son imágenes mentales. De hecho, parecería

14 Locke, John. op. cit. II, xxiii, §2. La cursiva és de Locke. La traducción al castellano es mía; la cita original dice así: «The Idea then we have, to which we give the general name Substance, being nothing, but the supposed, but unknown support of those Qualities, we find existing, which we imagine cannot subsist, sine re substante, without something to support them, we call that Support Substantia; which, according to the true import of the Word, is in plain English, standing under, or upholding»..

15 En relación al argumento usado por Berkeley para probar la existencia de Dios véase: Oya, Alberto (2015). "Análisis del argumento de Berkeley para probar la existencia de Dios (Tratado sobre los principios del conocimiento humano I, §29)”. Endoxa: Series Filosóficas, n. 35, pp. 109-118.

16 Se trata de los argumentos desarrollados en Principios I, §4, I, §5-7 y I, §23. Para un análisis de estos argumentos véase: Oya, Alberto (2017). "George Berkeley: els arguments positius a favor del immaterialisme i el principi de semblança". Comprendre. Revista Catalana de Filosofia, vol. 19, n. 1, pp. 83-92.. 
que el núcleo fundamental del argumento de Berkeley es que, una vez aceptado este supuesto fundamental de la epistemología moderna, no queda espacio para aceptar que una idea pueda representar algo completamente diferente a ella -que es lo que debería aceptarse si el representacionalismo de Locke fuera cierto. Es en este sentido que la concepción causal clásica del conocimiento perceptivo y el representacionalismo de John Locke pueden considerarse los puntos de partida del sistema filosófico berkelyano.

Por último, vale la pena, tal y como he hecho anteriormente, exponer la propuesta de Berkeley mediante un ejemplo breve y sencillo. Así, según Berkeley, cuando un individuo está percibiendo un árbol, lo que sucede son dos procesos simultáneos y diferentes: (i) una relación causal entre Dios, que es externo a la mente del sujeto, y la imagen interna de árbol que tiene el sujeto en su mente, en la cual Dios es la causa de la imagen interna del sujeto $\mathrm{y}$, por tanto, la segunda es el efecto del primero; (ii) una relación epistémica, por introspección, entre el sujeto y su imagen interna de árbol, en la cual el primero es el sujeto y la segunda el objeto.

\section{La crítica de Berkeley al representacionalismo}

En lo que queda de artículo ahondaré en la crítica de Berkeley al materialismo. Como ya dije, debe tenerse en cuenta que los argumentos ofrecidos por Berkeley en su Tratado sobre los Principios del Conocimiento Humano están especialmente dirigidos al materialismo representacionalista de John Locke.

La primera de las críticas de Berkeley consiste en la formulación del llamado «principio de semejanza». Y aunque la aceptación del principio de semejanza parecería comportar automáticamente el fracaso de cualquier proyecto representacionalista, en las secciones iniciales de la primera parte del Tratado sobre los Principios del Conocimiento Humano, Berkeley ofrece un conjunto de argumentos que son independientes de la validez del principio de semejanza y que pretenden atacar, de una manera u otra y por una u otra razón, el proyecto representacionalista.

\subsection{El principio de semejanza}

En $§ 8$ de la primera parte del Tratado sobre los Principios del Conocimiento Humano, Berkeley postula el que después ha sido conocido como «principio de semejanza», el cual constituye la principal crítica al supuesto representacionalista de que el contenido de nuestras ideas internas representa - esto es: se corresponde con- el mundo externo. El principio de semejanza consiste en la afirmación que entidades con un estatus ontológico distinto -mental y material en este caso- no pueden presentar relaciones de semejanza entre sí. Berkeley formula esta principio como sigue:

[...] una idea no puede parecerse a nada que no sea otra idea; un color o una figura sólo puede parecerse a otro color o figura. ${ }^{17}$

17 Berkeley, George. Treatise concerning the Principles of Human Knowledge. USA: Dover Publications, 2003. I, $\S 8$. La traducción al castellano es mía; la cita original dice así: «[...] an idea can be like nothing but an idea; a colour or figure can be like nothing but another colour or figure». 
Lo que está haciendo Berkeley con este principio es negar que algunas de nuestras ideas puedan representar algo que no sea una idea, que se encuentre fuera de la mente; y el representacionalismo se basa, precisamente, en el hecho de que todas o algunas de nuestras ideas -las ideas de cualidades primarias, en el caso de Lockesí representan verídicamente, mediante una relación de semejanza, cualidades que existen fuera de nuestra propia mente. Podría decirse, pues, que el principio de semejanza es la crítica principal al representacionalismo.

Aunque Berkeley no da, al menos explícitamente, ningún tipo de razón a favor del principio de semejanza, parece que hay una manera de justificarlo. La argumentación, que partiría de dos premisas aceptadas tanto por Berkeley como por Locke, puede ser formulada como sigue:

(1) Todo lo que percibimos por introspección son particulares mentales, ideas.

(2) Percibimos por introspección aquello que las ideas representan.

(3) Aquello que las ideas representan son rasgos de las propias ideas.

Y dado (3), es imposible que las ideas representen algo externo al sujeto, pues no hay ningún rasgo de las ideas que sea externo al sujeto.

Dejando de lado la cuestión por la plausibilidad de este principio, vale la pena apuntar que el buen punto de Berkeley al formularlo es que, dada la noción de idea que comparte con Locke, el principio de semejanza es un principio obligado para ambos. Y es que, independientemente de si es válido o no, el principio es importante por el hecho de que Berkeley lo acepta y que Locke parece estar obligado a aceptarlo, en tanto que acepta las premisas (1) y (2) de las que se deriva.

\subsection{Crítica a la distinción entre cualidades primarias y cualidades secundarias}

De ser cierto, el principio de semejanza supondría por sí mismo una refutación de la distinción entre cualidades primarias y cualidades secundarias. Aún así, Berkeley, en las secciones inmediatamente posteriores a la formulación del principio de semejanza, ${ }^{18}$ ofrece dos críticas a la distinción entre cualidades primarias y cualidades secundarias que son independientes de la validez de dicho principio.

El primero de estos argumentos prueba el carácter no-independiente de las cualidades primarias. Es decir: que las cualidades primarias no pueden ser concebidas con independencia de las cualidades secundarias. Así, por ejemplo, nos es imposible concebir un objeto extenso pero sin color:

Pero deseo que cada uno reflexione y pruebe si puede, por medio de alguna abstracción del pensamiento, concebir la extensión y el movimiento de un cuerpo sin el resto de cualidades sensibles. Por mi parte, veo claramente que no está en mi poder el formar una idea de un cuerpo extenso y con movimiento, sino que debo darle también algún color o alguna otra cualidad sensible, las cuales se reconoce que existen sólo en la mente. Dicho brevemente: extensión, figura, y movimiento, abstraídas del resto de cualidades, son inconcebibles. ${ }^{19}$

Berkeley, G. op. cit. I, §9-15.

19 Berkeley, G. op. cit. I, §10. La traducción al castellano es mía; la cita original dice así: «But I desire any one 
Y el problema para el representacionalista viene dado porque éste postula que las cualidades primarias existen, en los objetos materiales, con independencia de las cualidades secundarias.

Aquí podría contestarse a Berkeley que su argumento está limitado al ámbito epistemológico; es decir: que sólo hace referencia a nuestra capacidad de conocer las cosas y no a como éstas son de hecho, pues que nos sea imposible concebir -esto es: formarnos una idea o imagen mental de- un objeto extenso sin color no implica necesariamente que sea imposible que exista de hecho un objeto extenso sin color.

Ahora bien, creo que la intención última de Berkeley es la de poner de manifiesto que el representacionalista necesita del poder concebir un objeto extenso sin color si es que cuando dice que el color -y, en general, el resto de cualidades secundariasexisten sólo en la mente, está diciendo algo que tiene sentido, significado. La razón es la siguiente: un objeto extenso sin color no es una idea -y es imposible que lo sea, en tanto que producir una idea es concebir o formarse una imagen mental y ya hemos visto que concebir tal cosa es imposible-; por tanto, cuando el representacionalista nos dice que el color no existe fuera de la mente no está refiriendo a ninguna idea, no nos está diciendo nada, sólo juega con las palabras.

Podría calificarse de reductiva la segunda de las críticas usadas por Berkeley con tal de refutar la distinción entre cualidades primarias y cualidades secundarias, pues pretende demostrar que el principal argumento empleado para probar que las cualidades secundarias existen sólo en la mente de aquel quien las percibe también prueba, con la misma fuerza, que las cualidades primarias sólo existen en la mente de quien las percibe. Más concretamente, que el argumento de la relatividad de la percepción es también aplicable a las cualidades primarias:

[...] a cualquiera que permitáis considerar estos argumentos que se piensa prueban de manera manifiesta que los colores y los sabores existen sólo en la mente, encontrará que estos argumentos pueden usarse con la misma fuerza para probar lo mismo respecto a la extensión, la figura y el movimiento..$^{20}$

Llegados a este punto, creo que vale la pena dejar de lado por un momento la crítica de Berkeley y ver con un poco más de detalle en qué consiste el argumento de la relatividad de la percepción y el uso que hace John Locke de él.

Siguiendo a Marc-Wogau, ${ }^{21}$ podemos formular la estructura del argumento de la relatividad de la percepción, en su formulación de la corriente de agua que parece ser fría a una mano y caliente a la otra, de la manera siguiente:

(1) Una misma cosa no puede tener simultáneamente dos cualidades incompatibles

(2) Las cualidades frío y caliente son incompatibles

to reflect and try whether he can, by any abstraction of thought, conceive the extension and motion of a body without all other sensible qualities. For my own part, I see evidently that it is not in my power to frame an idea of a body extended and moving, but I must withal give it some colour or other sensible quality which is acknowledged to exist only in the mind».

20 Berkeley, G. op. cit. I, §15. La traducción al castellano es mía; la cita original dice así: «[...] let any one consider those arguments which are thought manifestly to prove that colours and taste exist only in the mind, and he shall find they may with equal force be brought to prove the same thing of extension, figure, and motion».

${ }^{21}$ Marc-Wogau, Konrad. “The Argument from Illusion and Berkeley's Idealism”; en: Creery, Walter E. (ed.) George Berkeley: Critical Assessments. Londres: Routledge, 1991, pp. 328-337. 
(3) Una cualidad percibida, que es el contenido de la percepción, es real o existe; es decir: es en realidad una cualidad de la cosa percibida

(4) El agua que parece fría a una mano y caliente a la otra es el mismo e idéntico objeto

Una variante de este argumento, que corresponde a su formulación del caramelo que parece dulce a un individuo y salado a otro individuo, consistiría en substituir, junto con el hecho que «las cualidades sensibles que son percibidas en un objeto cambian, mientras que el objeto se mantiene sin cambios», las premisas (1) y (2) por:

(5) Si una cualidad es inherente a un objeto, el cambio en la cualidad implica el cambio en el objeto

Para solucionar la contradicción que surge - esto es: que la misma corriente de agua es fría y caliente al mismo tiempo, o que el mismo caramelo es dulce y salado al mismo tiempo-, Locke afirma que aquellas cualidades incompatibles que parecen poder existir simultáneamente en un mismo objeto, y/o aquellas cualidades en las que un cambio en ellas no comporta ningún cambio en los objetos de los cuales son rasgos o aspectos, tienen un carácter interno o mental y que no representan verídicamente nada fuera de la mente. ${ }^{22}$ Dicho de manera más breve: son entidades mentales que no existen como propiedades de un objeto externo.

En mi opinión, es importante destacar que la respuesta de Locke no puede ser afirmar simplemente, por un lado, el carácter mental de una cualidad y negar, por otro lado, que ésta represente verídicamente nada fuera de la mente. Por si mismo esto no es una solución a la contradicción que surge del argumento de la relatividad de la percepción; pues lo único que comportaría es que dicha propiedad es un aspecto o rasgo de un objeto mental. Y que el objeto en cuestión sea mental no soluciona nada, pues éste sigue siendo una entidad particular, una imagen mental, completamente determinada. Es decir: es igual de contradictorio suponer un caramelo mental que es dulce y salado al mismo tiempo que un caramelo material que es dulce y salado al mismo tiempo. ${ }^{23}$

Esta objeción es, creo, totalmente válida si se toma la tesis de Locke aisladamente, sin tener en cuenta su teoría de las ideas simples como entidades independientes. En el momento en que la tenemos en cuenta, la objeción que acabo de presentar desaparece. Y es que la objeción sólo tiene sentido si aceptamos que el contenido de nuestra percepción es, en el caso de la corriente de agua, «calor y frío al mismo tiempo y en el mismo objeto» -y, en el caso del caramelo, «dulce y salado al mismo tiempo y en el mismo objeto». Pero esto es, precisamente, lo que la teoría de las ideas simples de Locke niega: el contenido de nuestra percepción sería, en estos casos, doble: «calor» y «frío» al mismo tiempo y en el mismo objeto, en el primer caso; «dulce» y «salado» al mismo tiempo y en el mismo objeto, en el segundo. De

22 Es decir: que el contenido de nuestras ideas de dichas cualidades no se corresponde con nada fuera de la mente, que aunque nuestra mente sí proyecta dichas cualidades, dicha proyección es errónea.

23 Quizás podría responderse a todo esto que, en realidad, cuando se afirma que una cualidad es mental y no representa verídicamente nada fuera de la mente, lo que realmente se está diciendo es que dicha cualidad es una cualidad de un sujeto o mente, y no de un objeto mental. Esto, sin embargo, es absurdo: la mente mantiene cierta relación epistémica con las cualidades frío y caliente, dulce y salado; pero éstas no son predicados de la mente: la mente no es fría o caliente, dulce o salada. 
ser así la contradicción puesta de manifiesto por el argumento desaparece, ya que se acepta que percepción o idea de «calor», por un lado, y percepción o idea de «frío», por otro, son dos cosas o entidades diferentes e independientes; en pocas palabras: se niega la premisa (4) del argumento.

Dicho de otro modo: la contradicción no reside en el contenido de nuestras percepciones, sino en la forma en qué nuestra mente constituye los diferentes objetos que percibe -es decir: en la manera cómo la mente agrupa las diferentes ideas simples de percepción configurando un objeto. Pero, de acuerdo con Locke, la contradicción es ahora ya sólo aparente, en tanto que las diferentes ideas simples de percepción son entidades u objetos plenamente independientes las unas de las otras.

Ahora bien, el problema para Locke es, en mi opinión, que su teoría de las ideas simples como entidades independientes parece ser una descripción del contenido perceptivo, además de inverosímil, falsa. Esto es, de hecho, lo que parece probar el carácter no independiente que presentan nuestras ideas de cualidades: no podemos tener una percepción o idea de «calor», «frío», «dulce», «salado», etc., aisladamente -esto es: sin percibir al mismo tiempo que sean rasgos de un objeto particular, o percibiendo que sean las únicas cualidades de un objeto particular-, cosa que parecería que deberíamos poder hacer si la teoría de las ideas simples como entidades independientes de Locke fuera cierta.

Volviendo ya a la crítica que hace Berkeley de la propuesta de Locke, cabe decir que hay otra variante del argumento de la relatividad de la percepción que no necesita de la posibilidad de percibir propiedades incompatibles al mismo tiempo: la percepción de color depende exclusivamente de las peculiaridades de nuestro cuerpo y del contexto en qué nos encontramos -los cambios de luz, principalmente-; y si las ideas de color dependen del sujeto que las tiene y del contexto en qué éste se encuentra, diríamos que no hay nada en el objeto externo que sea parecido a nuestras ideas de color. Ahora bien, este argumento, como bien vio Berkeley, si demostrara alguna cosa, demostraría también la no existencia en los objetos externos de las llamadas cualidades primarias.

Así, por ejemplo, se dice que el calor y el frío son afecciones sólo en la mente, y que en ningún caso son modelos de seres reales que existen en las sustancias corpóreas que las excitan; y es por esto que el mismo cuerpo que se muestra frío a una mano parece caliente a la otra. Ahora bien, ¿por qué no podemos argumentar también que la figura y la extensión no son modelos, o que no se asemejan, a cualidades que existen en la materia, pues al mismo ojo en diferentes posiciones, o a ojos con diferentes texturas en la misma posición, figura y extensión aparecen de manera diferente y no pueden, en consecuencia, ser imágenes de nada establecido y determinado sin la mente? ${ }^{24}$

En relación a todo lo que hemos dicho aquí acerca del argumento de la relatividad de la percepción, parece que podemos concluir que la respuesta de Locke a dicho

24 Berkeley, G. op. cit. I, §14. La traducción al castellano es mía; la cita original dice así: «Thus, for instance, it is said that heat and cold are affections only of the mind, and not at all patterns of real beings, existing in the corporeal substances which excite them, for that the same body which appears cold to one hand seems warm to another. Now, why may we not as well argue that figure and extension are not patterns or resemblances of qualities existing in Matter, because to the same eye at different stations, or eyes of a different texture at the same station, they appear various, and cannot therefore be the images of anything settled and determinate without the mind?» 
argumento es por sí sola incapaz de solucionar la contradicción que surge a raíz del mismo, y que requiere del concurso de su teoría de las ideas simples como entidades independientes, teoría que es aceptada tanto por Locke como por Berkeley. Por otro lado, dicha teoría, además de inverosímil, parece ser falsa. En cualquier caso, cabe decir que, dada la estrategia reductiva aplicada por Berkeley también a las cualidades primarias, la tesis de Locke no es válida para justificar la distinción entre cualidades primarias y cualidades secundarias.

\subsection{La expresión 'sustancia material' carece de significado}

Con la expresión 'sustancia material', el materialista, dice Berkeley, parece significar la idea de Ser en general juntamente con la idea relativa de que es el sustrato que soporta las cualidades de los objetos. La crítica de Berkeley pretende probar que esta doble definición de sustancia material carece de significado, pues no refiere a ninguna idea.

Por un lado, el autor apunta que esta idea de Ser en general no es nada más que una idea abstracta, y Berkeley considera que las ideas abstractas son inconcebibles. Es decir: definir 'sustancia material' como la idea de Ser en general es no decir absolutamente nada, pues esta definición apela a una idea abstracta que, como tal, no existe. ${ }^{25}$

En la Introducción a su Tratado sobre los principios del conocimiento humano, Berkeley ofrece dos argumentos para probar que las ideas abstractas son inconcebibles. Si Berkeley está en lo cierto, dado que el ser de una idea es su ser percibido, debemos concluir que las ideas abstractas no existen.

El primer argumento dice así: sólo podemos concebir ideas determinadas, y las ideas abstractas son indeterminadas; por tanto, las ideas abstractas son inconcebibles. Berkeley justifica la primera premisa afirmando que es un hecho que cuando imaginamos un objeto cualquiera, éste tiene siempre unas propiedades claramente definidas. Así, por ejemplo, cuando imagino un hombre, la idea resultante de este proceso imaginativo es una imagen mental que representa un hombre particular con unas propiedades completamente determinadas -tiene los ojos, el cabello, la piel, etc., de una manera concreta, y no de otra. La segunda premisa de este primer argumento se justifica por la propia definición de idea abstracta: una idea abstracta, cualquiera que sea ésta, requiere de un cierto grado de indeterminación, pues debe poder ser válida para referir a un conjunto de particulares de una misma clase, los cuales no son exactamente idénticos entre sí, y no sólo a un único particular, que es lo que sucedería si la idea estuviera completamente determinada. ${ }^{26}$

25 Berkeley, G. op. cit. I, §17: «La idea de ser en general me parece ser la más abstracta e incomprensible de todas las ideas $[\ldots .]$.$» .La traducción al castellano es mía; la cita original dice así: «The general idea of Being appeareth$ to me the most abstract and incomprehensible of all other $[\ldots] »$.

26 Aquí es importante el «no son exactamente idénticos entre sí»: es posible que un conjunto de diferentes particulares compartan la misma cualidad particular -por ejemplo: que un bolígrafo y una camisa compartan el mismo (idéntico) color, que los dos sean de un color azul marino idéntico (por tanto, con el mismo grado de intensidad) - o que dos objetos particulares diferentes compartan exactamente todas las cualidades -por ejemplo: dos hombres particulares que sean exactamente idénticos. Es cierto, por tanto, que una idea de un objeto -o cualidad- completamente determinada puede referir a más de un particular. En cualquier caso, las ideas abstractas siguen requiriendo de cierto grado de indeterminación, pues los objetos que agrupa una idea abstracta, sea cual sea ésta, no son nunca exactamente idénticos. 
El segundo argumento de Berkeley es como sigue: el contenido de las ideas abstractas es lógicamente contradictorio; por tanto, las ideas abstractas son inconcebibles. Más concretamente: el contenido de las ideas abstractas viola el principio del tercio excluido. ${ }^{27} \mathrm{Y}$ esto es lo que nos quiere hacer ver Berkeley con el caso de la idea abstracta de triángulo:

¿Qué puede ser más fácil para usted que mirar un poco dentro de sus propios pensamientos, e intentar entonces descubrir si tiene, o puede llegar a tener, una idea que se corresponda con la descripción que se ha dado aquí de la idea general de triángulo, la cual no es «ni oblicuo, ni rectangular, tampoco equilátero, isósceles, ni escaleno, pero todos y ninguno de estos al mismo tiempo»? ${ }^{28}$

Y es que la idea abstracta de triángulo debería de representar un triángulo que fuera y no fuera, al mismo tiempo, equilátero, isósceles, escaleno, obtuso y rectángulo, pues la idea abstracta de triángulo debe de satisfacer la descripción de todos los triángulos particulares existentes. La razón de esto es similar a la usada para justificar la segunda premisa del primero de los argumentos de Berkeley comentados aquí: una idea abstracta debe de poder ser válida para referir a todo el conjunto de particulares de una misma clase, y no sólo a un único particular. En el caso que nos ocupa: si la idea abstracta de triángulo correspondiera a una imagen mental de, por ejemplo, triángulo isósceles, entonces ya no sería válida para referir al conjunto de miembros que forman la clase triángulo, pues no satisfaría la descripción de los triángulos de tipo equilátero, escaleno, obtuso y rectángulo; $\mathrm{y}$, al revés, si no correspondiera a una imagen de triángulo isósceles tampoco sería válida para referir al conjunto de miembros que forman la clase triángulo, pues no satisfaría la descripción de los triángulos de tipo isósceles. De aquí la justificación que la idea abstracta de triángulo deba de ser y no ser, al mismo tiempo, todos los tipos de triángulos posibles.

La opinión que podemos formarnos ideas abstractas proviene, dice Berkeley, de una concepción equivocada del lenguaje. Por un lado, de la creencia que toda palabra tiene un único y exacto significado -es decir: que refiere a una única idea-; de esta creencia y del hecho que el lenguaje contiene términos generales, se deriva la existencia de ideas abstractas -los términos generales refieren a ideas abstractas. ${ }^{29} \mathrm{Y}$, por otro lado, de la creencia que toda palabra con significado designa una idea, junto con la creencia que la única finalidad del lenguaje es comunicar nuestras ideas; a partir de aquí y del hecho de que hay palabras que se presupone tienen significación pues sirven a la comunicación- pero no designan ninguna idea particular concebible, se llega a la conclusión de que estas palabras designan ideas abstractas. ${ }^{30}$

Respecto al primer caso, Berkeley afirma que es falso que a toda palabra le corresponda un único y exacto significado: los términos generales no significan ideas generales, sino cualquiera de las ideas particulares del mismo grupo que denotan. ${ }^{31}$

27 Este principio dice así: para cualquier objeto $x$ y cualquier propiedad $P$, o bien $x$ tiene $P$ o bien $x$ no tiene $P$.

28 Berkeley. G. op. cit., Intr., §13. La traducción al castellano es mía; la cita original dice así: «What more easy than for anyone to look a little into his own thoughts, and there try whether he has, or can attain to have, an idea that shall correspond with the description that is here given of the general idea of a triangle, which is "neither oblique nor rectangle, equilateral, equicrural, nor scalenon, but all and none of these at once?».

29 Berkeley. G. op. cit., Intr., §18.

30 Berkeley. G. op. cit., Intr., §19.

31 Berkeley. G. op. cit., Intr, §18: «[...] no hay, en realidad, algo así como una significación precisa y determinada 
Respecto al segundo, Berkeley argumenta que es falso que la única finalidad del lenguaje sea la de comunicar nuestras ideas, pues: en primer lugar, el lenguaje tiene otras finalidades, como «despertar alguna pasión, estimular o disuadir la realización de una acción, poner a la mente en cierta disposición particular»; ${ }^{32}$ en segundo lugar, no es necesario que las palabras despierten en nosotros las ideas que designan; ${ }^{33}$ en tercer lugar, hay palabras que no designan ninguna idea. ${ }^{34}$

Por otro lado, volviendo a la crítica de Berkeley a la noción de sustancia material, Berkeley se pregunta qué quiere decir el materialista cuando afirma que 'sustancia material' designa el substrato que sustenta las cualidades de los objetos. Parece claro que el materialista no está apelando aquí al sentido ordinario de la expresión 'que sustenta' - «como cuando decimos que los pilares sustentan el edificio», dice Berkeley ${ }^{35}$ sino a otro uso metafórico. Cuál es este uso metafórico, no obstante, es algo que ningún materialista acierta a explicar. Por tanto cuando el materialista dice que la sustancia material es aquello que sustenta las cualidades de los objetos, tampoco está diciendo nada.

Quizás podría objetarse aquí a Berkeley que la expresión 'sustancia espiritual', que constituye uno de los pilares fundamentales de su «esse est percipi aut percipere», presenta problemas similares. Sin embargo, nótese que esto no es del todo cierto: es verdad que, igual que sucede con el caso de la sustancia material, no tenemos ninguna idea positiva de sustancia espiritual; ahora bien, a diferencia de lo que sucede con la tesis materialista, Berkeley sí puede dar una explicación de cómo la sustancia espiritual 'sustenta' las cualidades percibidas -esto es: mediante relaciones de percepción.

anexa a ningún término general, significando todos ellos un gran número de ideas particulares de manera diferente» (La traducción al castellano es mía; la cita original dice así: «[...] in truth, there is no such thing as one precise and definite signification annexed to any general name, they all signifying indifferently a great number of particular ideas»)).

32 Berkeley. G. op. cit., Intr., §20. La traducción al castellano es mía; la cita original dice así: «[...] the raising of some passion, the exciting to or deterring from an action, the putting the mind in some particular disposition $[\ldots] \gg$.

33 Berkeley. G. op. cit., Intr., §19: «Y un poco de atención descubrirá que no es necesario (incluso en los razonamientos más rigurosos) que los términos significativos que significan ideas deban, cada vez que son usados, excitar en el entendimiento las ideas que significan -en la lectura y la discusión, los términos son en su mayor parte usados como las letras lo son en algebra, en la cual, aunque cada letra marca una cantidad particular, no es, sin embargo, un requisito para proceder correctamente el que en todos los pasos cada una de sus letras sugiera a nuestros pensamientos aquella cantidad particular que estaba designada a significar». (La traducción al castellano es mía; la cita original dice así: «And a little attention will discover that it is not necessary (even in the strictes reasonings) significant names which stand for ideas should, every time they are used, excite in the understanding the ideas they are made to stand for -in reading and discoursing, names being for the most part used as letters are in Algebra, in which, though a particular quantity be marked by each letter, yet to proceed right it is not requisite that in every step each letter suggest to your thoughts that particular quantity it was appointed to stand for»).

34 Berkeley. G. op. cit., Intr., §19: «Que entre los hombres especulativos hay muchos términos que no sugieren a los demás ideas particulares, determinadas, o, en realidad, nada en absoluto, es algo que nadie negará». (La traducción al castellano es mía; la cita original dice así: «That there are many names in use amongst speculative men which do not always suggest to others determinate, particular ideas, or in truth anything at all, is what nobody will deny»).

35 Berkeley, G. op. cit. I, §16. La traducción al castellano es mía; la cita original dice así: «as when we say that pillars support a building». 


\subsection{El representacionalismo conduce al escepticismo}

Otra de las críticas de Berkeley es que el representacionalismo, y, en general, el materialismo, conduce necesariamente al escepticismo. La idea principal es la siguiente. Aunque tomemos el representacionalismo como verdadero, nunca podremos explicar si -ni tampoco por qué o cuáles- algunas de nuestras ideas representan cualidades de los objetos materiales: ni la razón ni los sentidos, nuestras dos únicas facultades que nos permitirían explicar tal cosa, son capaces de dar una explicación. Este argumento es de vital importancia, pues lo que Berkeley está poniendo aquí de manifiesto es la imposibilidad de que el representacionalismo, y también el materialismo en general, sea demostrado.

Mediante los sentidos no podemos llegar a conocer la existencia de objetos materiales, pues, como ya dijimos en la sección anterior, los sentidos sólo nos proporcionan información sobre nuestras ideas, que son internas.

Por lo que refiere a nuestros sentidos, con ellos sólo llegamos al conocimiento de nuestras sensaciones, ideas, o aquellas cosas que son inmediatamente percibidas por los sentidos, llámeseles como se quiera; pero no nos informan de que existen cosas sin la mente, o nopercibidas, semejantes a aquellas que son percibidas. ${ }^{36}$

Mediante la razón -esto es: infiriendo la existencia de objetos materiales de aquello que percibimos de manera inmediata, las ideas-tampoco es posible, ya que no hay un vínculo causal necesario entre nuestras percepciones y el mundo material.

Queda, por tanto, que si de algún modo tenemos algún conocimiento de las cosas externas, éste debe de ser obtenido mediante la razón, infiriendo su existencia de aquello que es inmediatamente percibido por los sentidos. Pero, ¿qué razón puede inducirnos a creer en la existencia de cuerpos sin la mente, partiendo de aquello que percibimos, si los mismos partidarios de la materia no pretenden que haya ninguna conexión necesaria entre ellos y nuestras ideas? Afirmo que es algo admitido por todos (y lo que sucede en los sueños, alucinaciones y demás, lo pone fuera de toda discusión) que es posible que fuéramos afectados por todas las ideas que ahora tenemos aun cuando no hubieran cuerpos a los que nuestras ideas se asemejaran y que existieran fuera de la mente. Por tanto, es evidente que la suposición de cuerpos externos no es necesaria para la producción de nuestras ideas, pues se acepta que a veces son producidas, y que podrían posiblemente ser producidas siempre, en el mismo orden con el que las vemos ahora, sin su participación. ${ }^{37}$

36 Berkeley, G. op. cit. I, 18. La traducción al castellano es mía; la cita original dice así: «As for our senses, by them we have the knowledge only of our sensations, ideas, or those things that are immediately perceived by sense, call them what you will: but they do not inform us that things exist without the mind, or unperceived, like to those which are perceived».

37 Berkeley, G. op. cit. I, §18. La traducción al castellano es mía; la cita original dice así: «It remains therefore that if we have any knowledge at all of external things, it must be by reason, inferring their existence from what is immediately perceived by sense. But what reason can induce us to believe the existence of bodies without the mind, from what we perceive, since the very patrons of Matter themselves do not pretend there is any necessary connexion betwixt them and our ideas? I say it is granted on all hands (and what happens in dreams, phrensies, and the like, puts it beyond dispute) that it is possible we might be affected with all the ideas we have now, though there were no bodies existing without resembling them. Hence, it is evident the supposition of external bodies is not necessary for the producing our ideas; since it is granted they are produced sometimes, and might possibly be produced always in the same order, we see them in at present, without their concurrence». Nótese 
Dicho más claramente: nadie puede establecer ningún tipo de semejanza o parecido entre alguna de sus ideas (cualquiera) y un objeto material externo a su mente porque para poder hacer tal cosa debería estar en disposición de conocer ambas cosas directamente, sus ideas internas y el mundo externo, pero esto es algo que el propio representacionalista niega, pues acepta que sólo conocemos de forma inmediata nuestras propias ideas internas.

\subsection{EI representacionalismo no es la mejor explicación}

A pesar de todos los argumentos expuestos hasta ahora, dice Berkeley, quizás alguien aún podría creer que vale la pena adoptar la tesis materialista porque ésta supone la mejor explicación a la pregunta de cómo son producidas nuestras ideas.

Pues bien, dice Berkeley, esto es rotundamente falso: el materialismo no tan sólo no da ninguna explicación al fenómeno de la producción de nuestras ideas, sino que, además, genera multitud de nuevas cuestiones, la mayoría de las cuales, por las razones que ya hemos comentado, es por definición incapaz de contestar -por ejemplo: ¿nuestras ideas representan objetos externos?, ¿cómo se distinguen aquellas ideas causadas por un objeto externo de aquellas que percibimos cuando soñamos?, ¿es nuestro entendimiento material o espiritual?, etc.

[...] pues, aunque concedamos a los materialistas sus cuerpos externos, no estarán por ello, ellos mismos así lo confiesan, más cerca de saber cómo son producidas nuestras ideas; pues se consideran a sí mismos incapaces de comprender de qué manera un cuerpo puede actuar sobre un espíritu, o cómo es posible que haya de imprimir alguna idea en la mente. En consecuencia, es evidente que la producción de ideas o sensaciones en nuestras mentes no puede ser una razón de porque deberíamos de suponer la existencia de materia o sustancias corpóreas, pues se reconoce que sería igual de inexplicable con o sin esta suposición..$^{38}$

\section{Conclusión}

A lo largo de este artículo he analizado los distintos argumentos ofrecidos por Berkeley en su Tratado sobre los principios del conocimiento humano para criticar el materialismo representacionalista de John Locke. Las conclusiones a las que podemos llegar son las siguientes.

En primer lugar, que el principio de semejanza es la crítica principal al representacionalismo de Locke, pues éste niega el supuesto básico sobre el que se sustenta el proyecto de Locke. Dejando de lado si este principio es válido o no, el punto importante es que el principio de semejanza parece ser un principio obligado para Locke, en tanto que acepta las premisas de las que éste se deriva.

que Berkeley dice algo similar en Principios, I, §20.

38 Berkeley, G. op. cit. I, §19. La traducción al castellano es mía; la cita original dice así: «[...] for, though we give the materialists their external bodies, they by their own confession are never the nearer knowing how our ideas are produced; since they own themselves unable to comprehend in what manner body can act upon spirit, or how it is possible it should imprint any idea in the mind. Hence it is evident the production of ideas or sensations in our minds can be no reason why we should suppose Matter or corporeal substances, since that is acknowledged to remain equally inexplicable with or without this supposition». 
En segundo lugar, hemos visto que Berkeley ofrece dos críticas distintas a la distinción entre las ideas de cualidades primarias y las ideas de cualidades secundarias. Por un lado, Berkeley apunta al carácter no-independiente de las cualidades primarias. Esto es un problema para el representacionalista porque éste necesita del poder concebir un objeto con cualidades primarias aislado del resto de cualidades secundarias si es que cuando dice que las cualidades secundarias existen sólo en la mente de quien las percibe está diciendo algo que tiene significado. Por otro lado, Berkeley ofrece una crítica reductiva al uso que hace Locke del argumento de la relatividad de la percepción. En relación a esto, he mostrado que la tesis de Locke requiere del concurso de su teoría de las ideas simples como entidades independientes, teoría que es aceptada tanto por Locke como por Berkeley. $\mathrm{He}$ apuntado que dicha teoría parece ser falsa, pero que, en cualquier caso, dada la estrategia reductiva aplicada por Berkeley también a las cualidades primarias, la tesis de Locke no es válida para justificar la distinción entre cualidades primarias y cualidades secundarias.

En tercer lugar, he apuntado que el razonamiento de Berkeley para probar que la expresión 'sustancia material' carece de significado depende principalmente de la crítica de Berkeley a las ideas abstractas. He negado la posibilidad de aplicar este mismo razonamiento a la noción de 'sustancia espiritual' que Berkeley utiliza, pues Berkeley sí puede dar una explicación de cómo la sustancia espiritual sustenta las cualidades percibidas (mediante relaciones de percepción).

Por último, he comentado que el argumento usado por Berkeley para probar que el materialismo conduce al escepticismo se basa principalmente en el supuesto de que el individuo sólo mantiene una relación epistémica inmediata, por introspección, con sus propias ideas internas, que son imágenes mentales; supuesto que es compartido por Locke. 